Homology, Homotopy and Applications, vol. 19(2), 2017, pp.83-87

\title{
ON THE DIMENSION OF CLASSIFYING SPACES FOR FAMILIES OF ABELIAN SUBGROUPS
}

\author{
GED COROB COOK, VICTOR MORENO, BRITA NUCINKIS AND \\ FEDERICO W. PASINI
}

(communicated by Graham Ellis)

\begin{abstract}
We show that a finitely generated abelian group $G$ of torsionfree rank $n \geqslant 1$ admits an $(n+r)$-dimensional model for $E_{\mathfrak{F}_{r}} G$, where $\mathfrak{F}_{r}$ is the family of subgroups of torsion-free rank less than or equal to $r \geqslant 0$.
\end{abstract}

\section{Introduction}

In this note we consider classifying spaces $E_{\mathfrak{F}} G$ for a family of subgroups $\mathfrak{F}$ of $G$. We are particularly interested in the minimal dimension, $\operatorname{denoted} \operatorname{gd}_{\mathfrak{F}} G$, such a space can have.

Let $G$ be a group. We say a collection of subgroups $\mathfrak{F}$ is a family if it is closed under conjugation and taking subgroups. A $G$-CW-complex $X$ is said to be a classifying space $E_{\mathfrak{F}} G$ for the family $\mathfrak{F}$ if, for each subgroup $H \leqslant G, X^{H} \simeq\{*\}$ if $H \in \mathfrak{F}$, and $X^{H}=\varnothing$ otherwise.

The spaces $\underline{E} G=E_{\mathfrak{F}} G$ for $\mathfrak{F}=\mathfrak{F} i n$ the family of finite subgroups and $\underline{\underline{E}} G=E_{\mathfrak{F}} G$ for $\mathfrak{F}=\mathcal{V}$ cyc the family of virtually cyclic subgroups have been widely studied for their connection with the Baum-Connes and Farrell-Jones conjectures respectively. For a first introduction into the subject see, for example, the survey [3].

We consider finitely generated abelian groups $G$ of finite torsion-free rank $r_{0}(G)=$ $n$ and families $\mathfrak{F}_{r}$ of subgroups of torsion-free rank less than or equal to $r<n$. Note that for $r=0, \mathfrak{F}_{0}=\mathfrak{F}$ in and that it is a well known fact, see, for example, $[\mathbf{3}]$, that $\mathbb{R}^{n}$ is a model for $\underline{E} G$ and that $\operatorname{gd}_{\mathfrak{F}_{0}} G=n$. For $r=1, \mathfrak{F}_{1}=\mathcal{V} c y c$ and it was shown in $\left[\mathbf{5}\right.$, Proposition 5.13(iii)] that $\operatorname{gd}_{\mathfrak{F}_{1}} G=n+1$.

The main idea is to use the method developed by Lück and Weiermann [5] to build models of $E_{\mathfrak{F}_{r}} G$ from models for $E_{\mathfrak{F}_{r-1}} G$. We begin by recalling those results in [5] that we need for our construction. Let $\mathfrak{F}$ and $\mathfrak{G}$ be families of subgroups of a given group $G$ such that $\mathfrak{F} \subseteq \mathfrak{G}$.

Definition 1.1. [5, (2.1)] Let $\mathfrak{F}$ and $\mathfrak{G}$ be families of subgroups of a given group $G$ such that $\mathfrak{F} \subseteq \mathfrak{G}$. Let $\sim$ be an equivalence relation on $\mathfrak{G} \backslash \mathfrak{F}$ satisfying:

The first author was partially supported by EPSRC grant EP/N007328/1 and the fourth author gratefully acknowledges the support by the Italian National Group for Algebraic and Geometric Structures and Their Applications (GNSAGA - INDAM).

Received September 29, 2016; published on September 6, 2017.

2010 Mathematics Subject Classification: 55R35, 20J06, 18 G99.

Key words and phrases: classifying space for a family, abelian group.

Article available at http://dx.doi.org/10.4310/HHA.2017.v19.n2.a5

Copyright (C) 2017, International Press. Permission to copy for private use granted. 
- For $H, K \in \mathfrak{G} \backslash \mathfrak{F}$ with $H \leqslant K$ we have $H \sim K$.

- Let $H, K \in \mathfrak{G} \backslash \mathfrak{F}$ and $g \in G$, then $H \sim K \Longleftrightarrow g H g^{-1} \sim g K g^{-1}$.

Such a relation is called a strong equivalence relation. Denote by $[\mathfrak{G} \backslash \mathfrak{F}]$ the equivalence classes of $\sim$ and define for all $[H] \in[\mathfrak{G} \backslash \mathfrak{F}]$ the following subgroup of $G$ :

$$
N_{G}[H]=\left\{g \in G \mid\left[g H g^{-1}\right]=[H]\right\} .
$$

Now define a family of subgroups of $N_{G}[H]$ by

$$
\mathfrak{G}[H]=\left\{K \leqslant N_{G}[H] \mid K \in \mathfrak{G} \backslash \mathfrak{F},[K]=[H]\right\} \cup\left(\mathfrak{F} \cap N_{G}[H]\right) .
$$

Here $\mathfrak{F} \cap N_{G}[H]$ is the family of subgroups of $N_{G}[H]$ belonging to $\mathfrak{F}$.

Theorem 1.2. [5, Theorem 2.3] Let $\mathfrak{F} \subseteq \mathfrak{G}$ and $\sim$ be as in Definition 1.1. Denote by $I$ a complete set of representatives of the conjugacy classes in $[\mathfrak{G} \backslash \mathfrak{F}]$. Then the $G$-CW-complex given by the cellular $G$ push-out

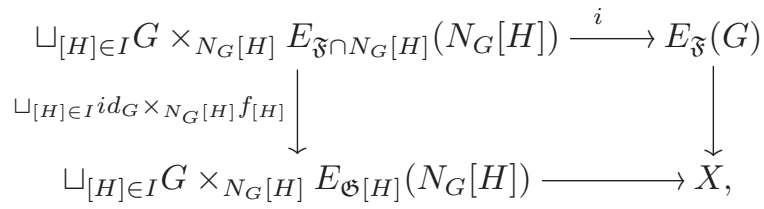

where either $i$ or the $f_{[H]}$ are inclusions, is a model for $E_{\mathfrak{G}}(G)$.

The condition on the two maps being inclusions is not that strong a restriction, as one can replace the spaces by the mapping cylinders, see [5, Remark 2.5]. Hence one has:

Corollary 1.3. [5, Remark 2.5] Suppose there exists an n-dimensional model for $E_{\mathfrak{F}} G$ and, for each $H \in I$, an $(n-1)$-dimensional model for $E_{\mathfrak{F} \cap N_{G}[H]}\left(N_{G}[H]\right)$ and an $n$-dimensional model for $E_{\mathfrak{G}[H]}\left(N_{G}[H]\right)$. Then there is an n-dimensional model for $E_{\mathfrak{G}} G$.

Corollary 1.3 gives us a tool to find an upper bound for $\operatorname{gd}_{\mathfrak{G}} G$. A very useful tool to find a lower bound for $\operatorname{gd}_{\mathfrak{G}} G$ is the following Mayer-Vietoris sequence [4], which is an immediate consequence of Theorem 1.2, see also [1, Proposition 7.1] for the Bredon-cohomology version.

Corollary 1.4. With the notation as in Theorem 1.2 we have following long exact cohomology sequence:

$$
\begin{aligned}
\cdots \rightarrow H^{i}\left(G \backslash E_{\mathfrak{G}} G\right) & \rightarrow\left(\prod_{[H] \in I} H^{i}\left(N_{G}[H] \backslash E_{\mathfrak{G}[H]} N_{G}[H]\right)\right) \oplus H^{i}\left(G \backslash E_{\mathfrak{F}} G\right) \rightarrow \\
& \prod_{[H] \in I} H^{i}\left(N_{G}[H] \backslash E_{\mathfrak{F} \cap N_{G}[H]} N_{G}[H]\right) \rightarrow H^{i+1}\left(G \backslash E_{\mathfrak{G}} G\right) \rightarrow \cdots
\end{aligned}
$$

This note will be devoted to proving the following Theorem:

Main Theorem. Let $G$ be a finitely generated abelian group of finite torsion-free rank $n \geqslant 1$, and denote by $\mathfrak{F}_{r}$ the family of subgroups of torsion-free rank less than or equal to $r \geqslant 0$. Then

$$
\operatorname{gd}_{\mathfrak{F}_{r}} G \leqslant n+r .
$$


The case for more general classes of groups $G$ is going to be dealt with, using different methods, by the second author in his Ph.D. thesis.

\section{The construction}

Throughout, let $G$ denote a finitely generated abelian group of torsion-free rank $r_{0}(G)=n$.

The idea is to construct models for $E_{\mathfrak{F}_{r}} G$ in terms of models for $E_{\mathfrak{F}_{r-1}} G$ using the push-out of Theorem 1.2 inductively. As a first step we shall define an equivalence relation in the sense of Definition 1.1.

Lemma 2.1. Let $\sim$ denote the following relation on $\mathfrak{F}_{r} \backslash \mathfrak{F}_{r-1}$ :

$$
H \sim K \Longleftrightarrow r k(H \cap K)=r .
$$

Then $\sim$ is a strong equivalence relation.

Proof. We show that $\sim$ is transitive: If $H \sim K$ and $K \sim L$, this implies that both $H \cap K$ and $K \cap L$ are finite index subgroups of $K$. Hence also $H \cap K \cap L$ is a finite index subgroup of $K$, and, in particular, of $K \cap L$ and thus of $L$. Hence $H \cap L$ is finite index in both $H$ and $L$. The rest is easily checked.

Definition 2.2. We say a subgroup $M$ of $G$ is maximal if it is not properly contained in a subgroup of $G$ of the same torsion-free rank as $M$.

Lemma 2.3. $G$ satisfies $\left(M_{\mathfrak{F}_{r-1} \subseteq \mathfrak{F}_{r}}\right)$, i.e. every subgroup $H \in \mathfrak{F}_{r} \backslash \mathfrak{F}_{r-1}$ is contained in a unique $H_{\max } \in \mathfrak{F}_{r} \backslash \mathfrak{F}_{r-1}$, which is maximal.

Proof. The existence follows from [6]. As regards uniqueness, suppose $H$ is included in two different maximal elements $K, L \in \mathfrak{F}_{r} \backslash \mathfrak{F}_{r-1}$ : then $H \leqslant K L$. Note that, since $H \sim L$ and $H \leqslant L$, it follows that $|L: H|<\infty$. Hence

$$
|K L: K|=|L: K \cap L| \leqslant|L: H|<\infty
$$

implies $K L \in \mathfrak{F}_{r} \backslash \mathfrak{F}_{r-1}$, contrary to the maximality of $K$ and $L$.

Note that we always have maximal elements in $\mathfrak{F}_{r} \backslash \mathfrak{F}_{r-1}$ as long as the ambient group is polycyclic [6], but uniqueness already fails for the Klein-bottle group $K$, which is non-abelian but contains a free abelian subgroup of rank 2 as an index 2 subgroup. Denote

$$
K=\left\langle a, b \mid a b a^{-1}=b^{-1}\right\rangle
$$

and consider $\mathfrak{F}_{1}$ the family of cyclic subgroups. Since $a^{2}=\left(a b^{-1}\right)^{2}$, in follows that $\left\langle a^{2}\right\rangle \leqslant\left\langle a b^{-1}\right\rangle$ as well as $\left\langle a^{2}\right\rangle \leqslant\langle a\rangle$, both of which are maximal.

For $M \leqslant G$ a subgroup of $G$ we denote by $\mathfrak{A} l l(M)$ the family of all subgroups of $M$.

Lemma 2.4. Let $M$ be a maximal subgroup of $G$ of torsion-free rank $r$. Then $\mathbb{R}^{n-r}$ is a model for $E_{\mathfrak{A l l}(M)} G$, and $\operatorname{gd}_{\mathfrak{A l l}(M)} G=n-r$.

Proof. Since $M$ is maximal it follows that $G / M$ is torsion-free of rank $n-r$ and hence $\mathbb{R}^{n-r}$ is a model for $E(G / M)$. The action of $G$ given by the projection $G \rightarrow G / M$ now yields the claim. 
Lemma 2.5. Let $\mathfrak{F}$ and $\mathfrak{G}$ be two families of subgroups of $G$. Then

$$
\operatorname{gd}_{\mathfrak{F} \cup \mathfrak{G}} G \leqslant \max \left\{\operatorname{gd}_{\mathfrak{F}} G, \operatorname{gd}_{\mathfrak{G}} G, \operatorname{gd}_{\mathfrak{F} \cap \mathfrak{G}} G+1\right\} .
$$

Proof. By the universal property of classifying spaces for families, there are maps, unique up to $G$-homotopy, $E_{\mathfrak{F} \cap \mathfrak{G}} G \rightarrow E_{\mathfrak{G}} G$ and $E_{\mathfrak{F} \cap \mathfrak{G}} G \rightarrow E_{\mathfrak{F}} G$. Now the double mapping cylinder yields a model for $E_{\mathfrak{F} \cup \mathfrak{G}} G$ of the desired dimension.

Lemma 2.6. Given $r<n$, suppose there exists a $d \geqslant n$ such that $\operatorname{gd}_{\mathfrak{F}_{r-1}} G \leqslant d$ and that for all maximal subgroups $N$ with $r_{0}(N)>r-1$ we also have $\operatorname{gd}_{\mathfrak{F}_{r-1} \cap \mathfrak{A l l}(N)} G \leqslant$ d. Then

$$
\operatorname{gd}_{\mathfrak{F}_{r}} G \leqslant d+1 \quad \text { and } \quad \operatorname{gd}_{\mathfrak{F}_{r} \cap \mathfrak{A} l l(M)} G \leqslant d+1
$$

for all maximal subgroups $M$ of $r_{0}(M)>r$.

Proof. We begin by applying Theorem 1.2 to the families $\mathfrak{G}=\mathfrak{F}_{r}$ and $\mathfrak{F}=\mathfrak{F}_{r-1}$. Lemma 2.3 implies that $G$ satisfies $\left(M_{\mathfrak{F}_{r-1} \subseteq \mathfrak{F}_{r}}\right)$. Denote by $\mathcal{N}$ the set of equivalence classes of maximal elements in $\mathfrak{F}_{r} \backslash \mathfrak{F}_{r-1}$. Then [5, Corollary 2.8] gives a push-out:

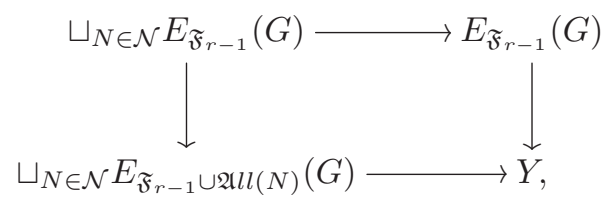

and $Y$ is a model for $E_{\mathfrak{F}_{r}} G$.

By assumption we have that $\operatorname{gd}_{\mathfrak{F}_{r-1}} G \leqslant d$ and $\operatorname{gd}_{\mathfrak{F}_{r-1} \cap \mathfrak{A} l l(N)} G \leqslant d$ for all $N \in \mathcal{N}$. Furthermore, by Lemma 2.4 we have that $\operatorname{gd}_{\mathfrak{A l l}(N)} G=n-r_{0}(N)<n$. Lemma 2.5 now implies that $\operatorname{gd}_{\mathfrak{F}_{r-1} \cup \mathfrak{A} l l(N)} G \leqslant d+1$. Applying Corollary 1.3 to the above pushout yields

$$
\operatorname{gd}_{\mathfrak{F}_{r}} G \leqslant d+1
$$

The second claim is proved similarly applying Theorem 1.2 to the families $\mathfrak{G}=$ $\mathfrak{F}_{r} \cap \mathfrak{A} l l(M)$ and $\mathfrak{F}=\mathfrak{F}_{r-1} \cap \mathfrak{A} l l(M)$. The argument of Lemma 2.3 applies here as well and hence $G$ satisfies $\left(M_{\left(\mathfrak{F}_{r-1} \cap \mathfrak{A} l l(M)\right) \subseteq\left(\mathfrak{F}_{r} \cap \mathfrak{A} l l(M)\right)}\right)$. We denote by $\mathcal{N}(M)$ the set of equivalence classes of maximal elements in $\mathfrak{F}_{r} \cap \mathfrak{A} l l(M) \backslash \mathfrak{F}_{r-1} \cap \mathfrak{A} l l(M)$. this now gives us a push-out:

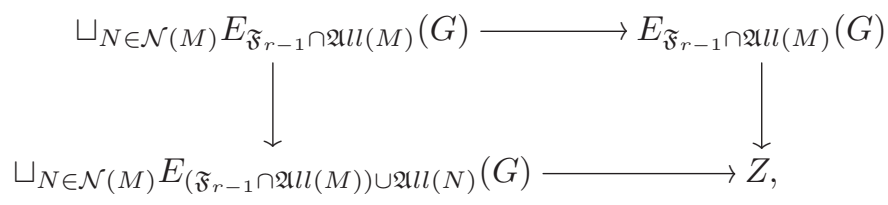

and $Z$ is a model for $E_{\mathfrak{F}_{r} \cap \mathfrak{A l l}(M)} G$.

Since $N \leqslant M$, it follows that $\left(\mathfrak{F}_{r-1} \cap \mathfrak{A} l l(M)\right) \cap \mathfrak{A} l l(N)=\mathfrak{F}_{r-1} \cap \mathfrak{A} l l(N)$ and hence, by assumption $\operatorname{gd}_{\left(\mathfrak{F}_{r-1} \cap \mathfrak{A} l l(M)\right) \cap \mathfrak{A} l l(N)} G \leqslant d$ and Lemma 2.5 implies that $\operatorname{gd}_{\left(\mathfrak{F}_{r-1} \cap \mathfrak{A} l l(M)\right) \cup \mathfrak{a l l ( N )}} G \leqslant d+1$. Now the same argument as above applies and

$$
\operatorname{gd}_{\mathfrak{F}_{r} \cap \mathfrak{A} l l(M)} G \leqslant d+1 .
$$

Proof of Main Theorem: We begin by noting that for $r=0$ we have that $\mathfrak{F}_{r}=\mathfrak{F}_{0}$ is the family of all finite subgroups of $G$. Then for all maximal subgroups $M$ of rank 1 , 
we have that $\mathfrak{F}_{0}=\mathfrak{F}_{0} \cap \mathfrak{A l l}(M)$. Furthermore, it is well known that $\operatorname{gd}_{\mathfrak{F}_{0}} G=n$, see, for example, $[\mathbf{3}]$.

Now an induction using Lemma 2.6 yields the claim.

Question 2.7. Is the bound of our Main Theorem sharp, i.e. for $n>r$, is

$$
\operatorname{gd}_{\mathfrak{F}_{r}} G=n+r ?
$$

Since $\operatorname{gd}_{\mathfrak{F}_{0}} G=\operatorname{gd}_{\mathfrak{F}_{0} \cap \mathfrak{A} l l(N)} G=n$ for all maximal subgroups $N$, we can assume equality in the inductive step (assumptions of Lemma 2.6). Then a successive application of the Mayer-Vietoris sequences to the push-outs in Lemmas 2.6 and 2.5, reduces the question to whether the map

$$
H^{d}\left(G \backslash E_{\mathfrak{F}_{r-1}} G\right) \rightarrow H^{d}\left(G \backslash E_{\mathfrak{F}_{r-1} \cap \mathfrak{A} l l(N)} G\right)
$$

is surjective or not.

We know by $[\mathbf{5}]$ that $\operatorname{gd}_{\mathfrak{F}_{1}} G=n+1$ and it was shown in $[\mathbf{2}]$ that the question has a positive answer for $G=\mathbb{Z}^{3}$, i.e. that $\operatorname{gd}_{\mathfrak{F}_{2}}\left(\mathbb{Z}^{3}\right)=5$.

\section{References}

[1] Dieter Degrijse and Nansen Petrosyan, Geometric dimension of groups for the family of virtually cyclic subgroups, J. Topol. 7 (2014), no. 3, 697-726, DOI 10.1112/jtopol/jtt045.

[2] A. Lopes-Onorio, Ph.D. thesis, University of Southampton (in preparation).

[3] W. Lück, Survey on classifying spaces for families of subgroups, Infinite Groups: Geometric, Combinatorial and Dynamical Aspects, 2005, pp. 269-322. MR2195456 (2006m:55036)

[4] _ Algebraische Topologie. Homologie und Mannigfaltigkeiten, Aufbaukurs Mathematik, Vieweg Studium, Wiesbaden, 2005.

[5] W. Lück and M. Weiermann, On the classifying space of the family of virtually cyclic subgroups, Pure Appl. Math. Q. 8 (2012), no. 2, 479-555.

[6] Daniel Segal, Polycyclic Groups, Cambridge Tracts in Mathematics, vol. 82, Cambridge University Press, Cambridge, 1983.

Ged Corob Cook ged.corobcook@cantab.net

Mathematics, University of Southampton, Southampton, SO17 1BJ, UK

Victor Moreno victormglezmoreno@gmail.com

Department of Mathematics, Royal Holloway, University of London, Egham, TW20 0EX, UK

Brita Nucinkis Brita.Nucinkis@rhul.ac.uk

Department of Mathematics, Royal Holloway, University of London, Egham, TW20 0EX, UK

Federico W. Pasini fpasini@uwo.ca

Department of Mathematics, University of Western Ontario, London, ON, N6A 5B7, Canada 